\title{
Actuator Fault Tolerant Control for a Rotary Wing Aircraft
}

\author{
Emre Kiyak* , Ahmet Ermeydan \\ Avionics Department,Faculty of Aeronauticsand Astronautics,EskisehirTechnicalUniversity, \\ Eskisehir, Turkey; *ekiyak@anadolu.edu.tr
}

SNE 28(3), 2018, 85 - 88, DOI: 10.11128/sne.28.sn.10422 Received: Sept. 15, 2016 (Selected EUROSIM Congress 2016 Postconf. Publ.), Revised July 30, Accepted: August 25, 2018 SNE - Simulation Notes Europe, ARGESIM Publisher Vienna, ISSN Print 2305-9974, Online 2306-0271, www.sne-journal.org

Abstract. Quadrotor is the one of the most popular rotary wing aircraft in control problems. There may be some problems in this system. In this case, quadrotor will not fly and do its duty effectively. In this study, a fault tolerant control strategy for a quadrotor is proposed in the presence of actuator faults. A linear mathematical model which is derived from a nonlinear model. The observer based state estimation approach is widely used in fault tolerant area. Here, this approach is used to detect and isolate faults in a quadrotor system. Generalized Observer Scheme (GOS) based Unknown Input Observer (UIO) is used for the isolation. Simulation results are presented to demonstrate the performance of the proposed fault tolerant control strategy.

\section{Introduction}

Mini-flying vehicles with four rotors which are called quadcopters have attracted many researchers in recent years. Vertical Take Off and Landing (VTOL), high maneuverability capabilities and indoor/outdoor navigation features make them popular. Besides that, by developing technology, especially in sensor devices, it is easy to obtain one of those vehicles. Therefore, a lot of studies implemented on these vehicles can be found in the literature. Many of them are generally related to different types of linear and nonlinear control techniques such as LQR (Bouabdallah et al, 2004), PID (Hoffman et al, 2010), backstepping (Madani and Benallegue, 2007) and nonlinear $\mathrm{H} \infty$ (Raffo et al, 2010) since quadcopters need autonomous stabilization systems to fly unlike airplanes. All of these works are focusing on healthy quadrotor systems. When a fault occurs in any of actuators, reliability should be maintained by detecting and isolating fault and modifying control scheme according to that fault case which is called fault tolerant control. There are various types of papers which present different solutions to this problem.

Sadeghzadeh et al. propose an active fault tolerant control method based on gain-scheduled PID in the presence of actuator faults for a quadrotor. An actuator fault detection system is presented using Thau's observer approach (Sadeghzadeh et al, 2012).

$\mathrm{Yu}$ et al. make a comparison between linear quadratic regulator and model predictive control for the height control of quadrotor with and without actuator faults (Yu et al, 2013).

A fuzzy gain-scheduled PID structure is proposed for the fault-tolerant control of a quadrotor when actuator faults occur (Amoozgar et al, 2012).

$\mathrm{He}$ and Zhao propose an Internal Model Control (IMC) design method based on quasi-LPV (Linear Parameter Varying) system. In their method, the nonlinear model is firstly transformed to the linear model based on the quasi-LPV method; then nonlinear motion function of the quadrotor is transformed to a transfer function matrix based on the transformation model from the state space to the transfer function. The performance of the controller proposed is tested by tracking for three reference trajectories with drastic changes and the simulation results indicate that the proposed control method has stronger robustness to parameters uncertainty and disturbance rejection performance (He and Zhao, 2015).

In this study, the problem of actuator fault detection, isolation and reconfiguration is addressed by an active fault tolerant method for a quadrotor. In detection and isolation part, the UIO method is used to find which actuator is faulty. In reconfiguration part, LQT switches to LQR with integral action to remove the steady-state error. The simulation study illustrates that the proposed actuator fault detection and isolation scheme is capable of detecting and identifying actuator failures in a variety of situations. The actuator faults are correctly detected and isolated for different conditions. 


\section{UIO Theory}

There are several model-based fault diagnosis methods in the literature, such as Kalman Filter based stochastic approach, observer-based approach, parity relation approach, parameter estimation approach. This study focuses on the UIO based fault detection and isolation of actuator faults. The main idea behind UIO approach is to estimate the state variables of the system utilizing mathematical model for generating residuals by subtracting the measured states from the estimated ones. In the fault isolation process, GOS is used to isolate the faulty actuator. This scheme consists of a bank of observers designed to make each residual to be sensitive to faults in all but one of the actuators.

The state space representation of a linear timeinvariant system with unknown input vector $d(t) \in$ $\mathbb{R}^{\mathrm{q} \times 1}$ can be described as following (Chen and Patton, 1999):

$$
\begin{aligned}
\dot{\mathrm{x}}(\mathrm{t})=\mathrm{Ax}(\mathrm{t}) & +\mathrm{Bu}(\mathrm{t})+\mathrm{Ed}(\mathrm{t}), \\
\mathrm{y}(\mathrm{t}) & =\mathrm{Cx}(\mathrm{t}),
\end{aligned}
$$

where $\mathrm{x}(\mathrm{t}) \in \mathbb{R}^{\mathrm{nx} 1}$ is the state vector, $\mathrm{u}(\mathrm{t}) \in \mathbb{R}^{\mathrm{mx} 1}$ is the input vector, $y(t) \in \mathbb{R}^{\mathrm{nx} 1}$ is the output vector of the aircraft model, $A \in \mathbb{R}^{\mathrm{nxn}}$ is system matrix, $B \in \mathbb{R}^{\mathrm{nxm}}$ is control distribution matrix, $E \in \mathbb{R}^{\text {nxq }}$ is unknown input distribution matrix and $C \in \mathbb{R}^{\mathrm{nxn}}$ is measurement distribution matrix.

UIO structure can be defined as follows:

$$
\begin{gathered}
\dot{\mathrm{z}}(\mathrm{t})=\mathrm{Fz}(\mathrm{t})+\mathrm{TBu}(\mathrm{t})+\mathrm{Ky}(\mathrm{t}), \\
\hat{\mathrm{x}}(\mathrm{t})=\mathrm{z}(\mathrm{t})+\mathrm{Hy}(\mathrm{t}),
\end{gathered}
$$

where $\hat{x}(t) \in \mathbb{R}^{n x 1}$ is the estimated state vector, $z(t) \in \mathbb{R}^{n x q}$ is the observation vector and $F, T, K, H \in$ $\mathbb{R}^{n x n}$ are the matrices to be designed so that error vector converges to zero. The error vector is defined as:

$$
e(t)=x(t)-\hat{x}(t)
$$

Provided that $\hat{\mathrm{x}}(\mathrm{t})$ in (5) is replaced by (4), the following yields:

$$
e(t)=(I-H C) x(t)-z(t) .
$$

Provided that the following conditions are satisfied and eigenvalues of the matrix $\mathrm{F}$ are selected in the open left half of the complex plane, the error goes to zero asymptotically:

$$
\begin{gathered}
(\mathrm{HC}-\mathrm{I}) \mathrm{E}=0, \\
\mathrm{~T}=\mathrm{I}-\mathrm{HC}, \\
\mathrm{F}=\mathrm{A}-\mathrm{HCA}-\mathrm{K}_{1} \mathrm{C}, \\
\mathrm{K}_{2}=\mathrm{FH}, \\
\mathrm{K}=\mathrm{K}_{1}+\mathrm{K}_{2} .
\end{gathered}
$$

To construct robust actuator fault isolation scheme, all sensors are accepted to be fault-free and system equation can be written as:

$$
\begin{gathered}
\dot{x}(t)=A x(t)+B^{i} u^{i}(t)+B^{i} f_{a}^{i}(t)+E^{i} d^{i}(t) \\
y=C x(t)
\end{gathered}
$$

where $b_{i} \in \mathbb{R}^{n}$ is the $i_{\text {th }}$ column of the matrix $B$, $B_{i} \in \mathbb{R}^{\mathrm{nx}(\mathrm{m}-1)}$ is obtained from the matrix $\mathrm{B}$ by deleting the $\mathrm{i}_{\text {th }}$ column $\mathrm{b}_{\mathrm{i}}, \mathrm{u}_{\mathrm{i}}$ is the $\mathrm{i}_{\mathrm{th}}$ component of $\mathrm{u}, \mathrm{u}_{\mathrm{i}} \in \mathbb{R}^{\mathrm{m}-1}$ is obtained from vector $u$ by deleting the $i_{\text {th }}$ component $\mathrm{u}_{\mathrm{i}}$, and

$$
E^{i}=\left[\begin{array}{ll}
E & b_{i}
\end{array}\right] ; d^{i}(t)=\left[\begin{array}{c}
d(t) \\
u_{i}(t)+f_{a i}(t)
\end{array}\right] .
$$

Based on the description above, UIOs and residual generators can be written as:

$$
\begin{gathered}
\dot{z}^{i}(t)=F^{i} z^{i}(t)+T^{i} B^{i} u^{i}(t)+K^{j} y(t) \\
r^{i}(t)=\left(I-C H^{i}\right) y(t)-C z^{i}(t) ; \quad \mathbf{l}=1,2 \ldots \mu
\end{gathered}
$$

\section{Equations Modelling of Quadrotor}

In this section, the nonlinear dynamics of the quadrotor is introduced and its linearization around equilibrium point is presented. Quadcopter movements, forces and coordinate frames are shown in Figure 1. $\left(\mathrm{x}_{\mathrm{i}}, \mathrm{y}_{\mathrm{i}}, \mathrm{z}_{\mathrm{i}}\right)$ and $\left(\mathrm{x}_{\mathrm{b}}, \mathrm{y}_{\mathrm{b}}, \mathrm{z}_{\mathrm{b}}\right)$ denotes inertial frame and body-fixed frame, respectively. $(\psi, \theta, \phi)$ refer to yaw, pitch and roll, respectively (Bresciani, 2008).

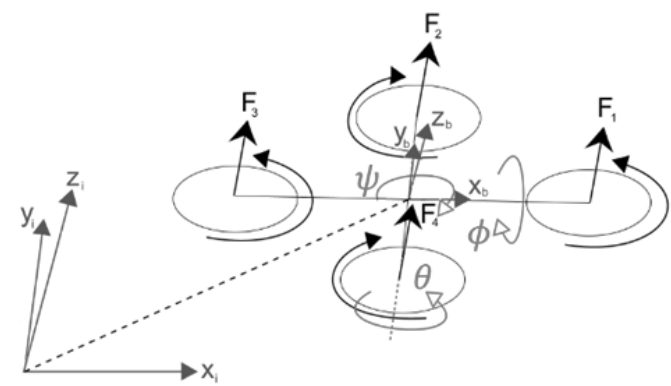

Figure 1. Quadrotor coordinate system. 
A nonlinear system can be expressed as follows:

$$
\dot{\mathbf{x}}=\mathrm{f}(\mathbf{x}, \mathbf{u})
$$

The state vector is chosen as following:

$$
\mathbf{x}=\left[\begin{array}{lllll}
x & y & z
\end{array} \theta \psi \dot{x} \dot{y} \dot{z} \dot{\phi} \dot{\theta} \dot{\psi}\right] \text {. }
$$

Since the quadrotor system is underactuated, four inputs can be defined as:

$$
\begin{gathered}
u_{1}=F_{1}+F_{2}+F_{3}+F_{4}, \\
u_{2}=l\left(F_{2}-F_{4}\right), \\
u_{3}=l\left(F_{1}-F_{3}\right), \\
u_{4}=\frac{d}{b}\left(F_{1}-F_{2}+F_{3}-F_{4}\right),
\end{gathered}
$$

where $b$ is thrust factor $\left(\mathrm{Ns}^{2}\right), d$ drag factor $\left(\mathrm{Nms}^{2}\right), l$ distance between propeller and center of gravity of quadrotor (m).

In this study, firstly, a quadrotor model is obtained in the simulation as seen Figure 2. Table 1 and 2 show gains for $\mathrm{X}, \mathrm{Y}$ and $\mathrm{Z}$ axis. Table 2 shows PID gains for $\mathrm{Z}$ axis.

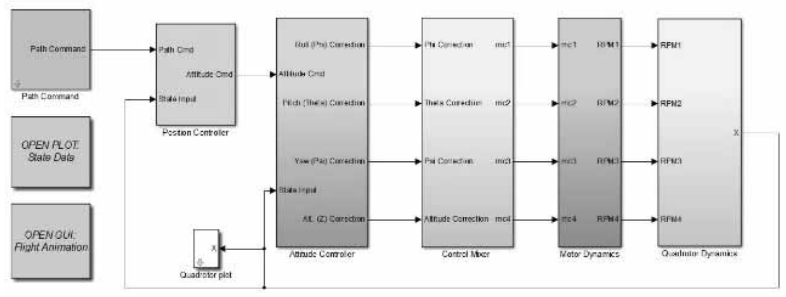

Figure 2. Nonlinear model of the quadrotor.

\begin{tabular}{|l|l|l|}
\hline & P & D \\
\hline X controller & 0.48 & 0.1 \\
\hline Y controller & 0.36 & 0.05 \\
\hline
\end{tabular}

Table 1. PD Gains for $X$ and $Y$ Axis.

\begin{tabular}{|l|l|l|l|}
\hline & P & I & D \\
\hline Z controller & 50 & 8 & 35 \\
\hline
\end{tabular}

Table 2. PID Gains for Z Axis.

Improved PID structure is proposed in Figure 3 for control. This PID structure presents the derivative action directly and prevents integral wind-up by limiting its maximum and minimum values. Saturation is introduced to integral part of PID controller defining a minimum and maximum value of which integral cannot go out. So it restores its linear behavior quickly.

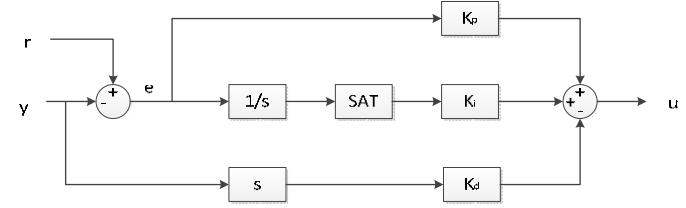

Figure 3. Improved PID structure.

\section{Results and Discussions}

It can be seen from the Figure 4 that LQT response is faster than LQR with integral action as expected due to integral effect but in the faulty case which is $10 \%$ throttle loss injected in $7 \mathrm{sec}$ of simulation time, the quadcopter with LQT controller tracks the given $\mathrm{X}$ and $\mathrm{Z}$ references with steady-state error. In contrary, LQR with integral action compensates steady-state error quickly. Y state is unaffected by the fault because the mathematical model used in the simulation is linear, so there is no coupling between channels.
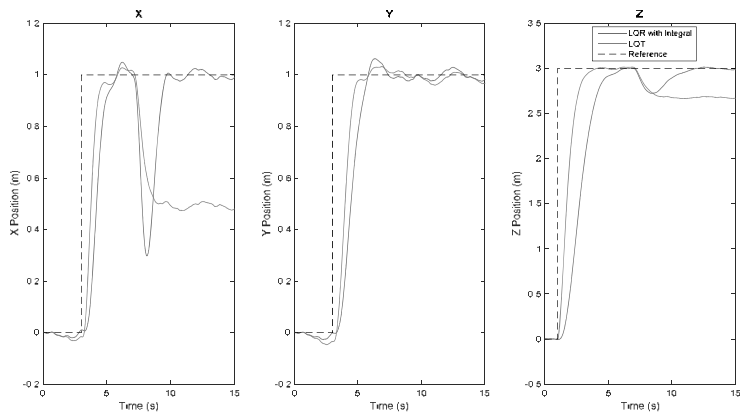

Figure 4. $X, Y$ and $Z$ response of LQT and LQR with integral with $10 \%$ fault on motor 1 .

The fault applied in the system is detected by UIO scheme. A bank of observers is designed based on GOS to isolate the fault in motor 2. Each observer is sensitive to a certain group of faults except one actuator. So motor 1 residual is nearly zero but remaining residuals are fired as presented in Figure 5.

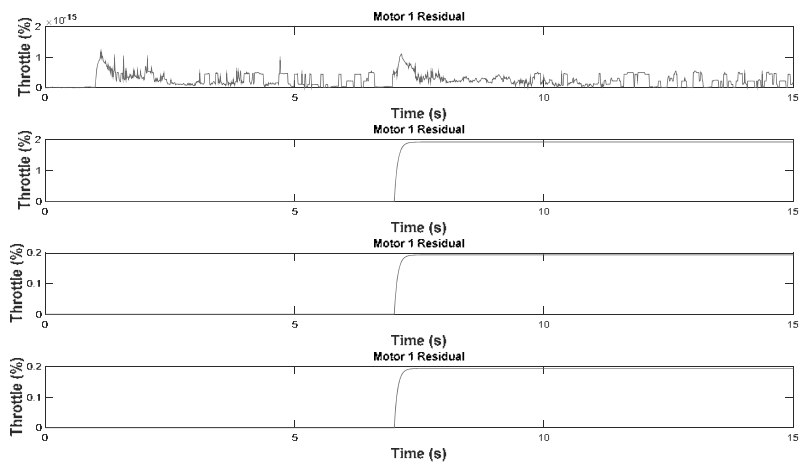

Figure 5. Residuals generated by UIO. 
After decision making by considering the structured residuals, LQR with integral action is engaged in the simulation instead of LQT. It can be easily seen in Figure 6 that the steady-state error is compensated quickly.
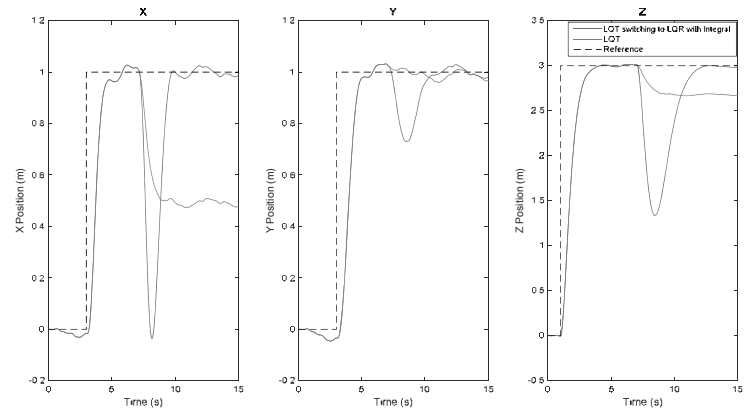

Figure 6. Height response of switching structure with $10 \%$ throttle fault on motor 1 .

\section{Conclusion}

In this study, a fault tolerant control method is introduced for the actuator faults in the quadrotor vehicle. The linear model of the quadrotor is derived from nonlinear equations. UIO is used as a method of detecting the fault and GOS to isolate it. Since LQT has no steady-state error in normal operation condition, there is no need to include integral effect when there is no fault. When it comes to faulty cases, it is seen that LQT could not compensate steady-state error. To eliminate the steady-state error, switching from LQT to LQR with integral action is performed. Results show that the proposed method represents fast response combining with the elimination of the error.

\section{References}

[1] Amoozgar MH, Chamseddine A, Zhang Y. FaultTolerant Fuzzy Gain-Scheduled PID for a Quadrotor Helicopter Testbed in the Presence of Actuator Faults, IFAC Conference on Advances in PID Control, Brescia, Italy, 2012.

[2] Bouabdallah S, Noth A, Siegwart R. PID vs LQ Control Techniques Applied to an Indoor Micro Quadrotor, IEEE/RSJ International Conference on Intelligent Robots and Systems, Vol. 3, pp.2451-2456, 2004.

[3] Bresciani T. Modelling, identification and control of a quadrotor helicopter, Msc Thesis, Lund University, Sweden. 2008.

[4] Chen J, Patton R. Robust Model-Based Fault Diagnosis for Dynamic Systems, Kluwer Academic Publishers, 1999.

[5] He Z, Zhao L. Quadrotor Trajectory Tracking Based on Quasi-LPV System and Internal Model Control, Mathematical Problems in Engineering, vol. 2015, pp.1-13, 2015.

[6] Henriques BSM. Estimation and Control of a Quadrotor Attitude, Msc Thesis, Universidade Tecnica de Lisboa, Portugal. 2011.

[7] Hoffman F, Goddemeier N, Bertram T. Attitude Estimation and Control of a Quadrocopter, IEEE/RSJ International Conference on Intelligent Robots and Systems, pp.1072-1077. 2010.

[8] Madani T, Benallegue A. Backstepping Control with Exact 2-Sliding Mode Estimation for a Quadrotor Unmanned Aerial Vehicle, IEEE/RSJ International Conference on Intelligent Robots and Systems, pp.141-146. 2007.

[9] Raffo GV, Ortega MG, Rubio FR. An Integral Predictive/Nonlinear $H_{\infty}$ Control Structure for a Quadrotor Helicopter, Automatica, Vol. 46, pp.29-39, 2010.

[10] Sadeghzadeh I, Mehta A, Chamseddine A, Zhang Y. Active Fault Tolerant Control of a Quadrotor UAV Based On Gain-Scheduled PID Control, 25th IEEE Canadian Conference on Electrical and Computer Engineering, pp.1-4. 2012.

[11] Yu B, Zhang Y, Minchala I, Qu Y. Fault-tolerant Control with Linear Quadratic and Model Predictive Control Techniques Against Actuator Faults in a Quadrotor UAV, Conference on Control and Fault-Tolerant Systems, France, pp.661-666, 2013. 